\title{
Random and Deterministic Perturbation of a Class of Skew-Product Systems
}

\author{
David Broomhead, Demetris Hadjiloucas, and Matthew Nicol \\ Dept of Mathematics, UMIST \\ Manchester \\ M60 1QD, UK.
}

August 20, 1999

\begin{abstract}
This paper concerns the stability properties of skew-products $T(x, y)=(f(x), g(x, y))$ in which $(f, X, \mu)$ is an ergodic map of a compact metric space $X$ and $g: X \times \mathbf{R}^{n} \rightarrow$ $\mathbf{R}^{n}$ is continuous. We assume that the skew-product has a negative maximal Lyapunov exponent in the fibre.

We study the orbit stability and stability of mixing of $T(x, y)=(f(x), g(x, y))$ under deterministic and random perturbation of $g$. We show that such systems are stable in the sense that for any $\epsilon>0$ there is a pairing of orbits of the perturbed and unperturbed system such that paired orbits stay within a distance $\epsilon$ of each other except for a fraction $\epsilon$ of the time.

We also show that such systems preserve ergodicity and higher order mixing properties under deterministic perturbation of $g$ and perturbation of $g$ by i.i.d. additive noise. Furthermore we show that the invariant measure for the perturbed system is continuous (in the Hutchinson metric) as a function of the size of the perturbation to $g$ (Lipschitz topology) and the noise distribution. Our results have applications to the stability of Iterated Function Systems which "contract on average".
\end{abstract}

\section{Introduction}

In this paper we address various questions to do with the stability of dynamically invariant subspaces. An obvious and topical application of our results is in the area of chaotic synchronisation [27] and its use in communication systems [12, 19]. Consider, for example, a dynamical system which has been divided into 2 parts:

$$
\frac{d}{d t}\left(\begin{array}{c}
v \\
w
\end{array}\right)=\left(\begin{array}{c}
h_{v}(v, w) \\
h_{w}(v, w)
\end{array}\right)
$$

where $(v, w) \in \mathbf{R}^{m+n}$. A simple arrangement for studying chaotic synchronisation is to force a second system

$$
\frac{d}{d t} w^{\prime}=h_{w}\left(v, w^{\prime}\right)
$$

with a time dependent input, $v$, found by solving equation (1.1). The dynamical system consisting of equation (1.1) and equation (1.2) is in the form of a skew product. The interest as far as chaotic synchronisation is concerned is to compare the quantities $w$ 
and $w^{\prime}$. In the simplest situation, synchronisation is associated with the existence of an invariant subspace in the skew product system which is stable in some sense. In this case the communications engineer has the luxury of having 2 synchronised (chaotic) oscillators-one in the transmitter and one in the receiver-which can be used to code and decode information.

More generally dynamically invariant subspaces often arise as a result of symmetries and in models displaying basin riddling or intermittent behaviour $[4,5,1,24,25,26,6]$. They play an organisational role and the dynamics restricted to the invariant subspace may drastically affect the dynamics of the ambient system. Recently their behaviour under additive noise has been investigated by several researchers $[11,28,34,3,9,13]$. In control theory and engineering the situation is often encountered in which the dynamics of a system restricted to an invariant subspace is an equilibrium state (synchronous coupled oscillators are a good example) and one is typically interested in the stability of the system to perturbations (random or deterministic) out of the invariant subspace. This is certainly true in the case of chaotic synchronisation where in any realistic communications application the channel transmitting the synchronising signal, $v$, would be distorted or noisy.

To be more specific we shall study skew product systems of the form: $T(x, y)=$ $(f(x), g(x, y))$ in which $(f, X, \mu)$ is an ergodic map of a compact metric space $X$ and $g: X \times \mathbf{R}^{n} \rightarrow \mathbf{R}^{n}$ is continuous. There are two distinct situations. If the dynamics is uniformly contracting onto the invariant subspace then large excursions away from the invariant subspace cannot be induced by perturbing $g$ deterministically or even randomly by additive noise. However, if the contraction is not uniform then arbitrarily large excursions from the invariant subspace are possible. Furthermore, attractors for the skew product system will not, in general, be continuous in the Hausdorff metric as a function of the perturbation. This contrasts with the uniformly contracting situation where it is easy to show that attractors are continuous in the Hausdorff metric, see for example [7, Theorem 11.1, page 111].

In the nonuniform case - where there is "contraction on average" $[14,15,2]$ (that is, a negative maximal Lyapunov exponent in the fibre, see Section 2 for a precise definition) - less is known about the stability of the dynamics under additive noise. Arnold and Crauel have shown the stability in the weak topology of invariant measures for such systems in the case that $g(x, y)$ is an affine mapping [2]. In the context of chaotic synchronisation, we might suppose that continuity of the attractor is necessary to ensure that the chaotic oscillator at the transmitter and its synchronised analogue at the receiver are close, given that the communication channel introduces small distortions and weak noise. In fact we shall show that this condition is not essential.

In this paper we show that if the function $g$ of a skew product $T(x, y)=(f(x), g(x, y))$ which has a negative maximal Lyapunov exponent in the fibre is perturbed to another function $\tilde{g}$ sufficiently close in the Lipschitz topology then the resulting skew product $\tilde{T}(x, y)=(f(x), \tilde{g}(x, y))$ preserves the mixing properties (ergodicity, strong mixing, Bernoulliness) of $T(x, y)$ (which are those of the base transformation). Similarly if $T(x, y)=(f(x), g(x, y))$ is perturbed by i.i.d. additive noise in the fiber then the mixing properties of the base transformation are inherited by the skew-product. In these cases (and in the more general case where the noise is not assumed i.i.d. ) given $\epsilon>0$, if the level of perturbation is sufficiently small then the orbits of the perturbed and unperturbed systems stay within $\epsilon$ of each other except for, at most, a fraction $\epsilon$ of the time. In general, if $\tilde{f}$ is a perturbed map of $f$, we say that the orbits of the perturbed 
and unperturbed maps stay within $\epsilon$ of each other except for at most a fraction $\epsilon$ of the time if

$$
\lim _{N \rightarrow \infty} \frac{1}{N}\left\{\# k:\left|f^{k}(x)-\tilde{f}^{k}(x)\right|>\epsilon, 1 \leq k \leq N\right\} \leq \epsilon .
$$

Our work is related to that of Campbell, Davies and Stark. Stark has investigated the existence and regularity properties of invariant graphs for skew-products in the papers [32, 33] and has uncovered interesting implications of such regularity for such phenomena as strange nonchaotic attractors. In particular, we have used certain results in [32, Section 5] in an essential way. Campbell and Davies [10] also have related results on the ergodic properties of attracting graphs and stability results for such graphs under deterministic perturbations. Campbell [9] considers the effect of noise in the slaved subsystem in a skew product system and obtains (under additional assumptions) bounds on the asymptotic size of the noise in terms of the behaviour of the forcing system. In particular Theorem 3.1 removes the assumption of Campbell on the existence of an absorbing set and in addition proves continuity of the invariant measure for the perturbed process in the Hutchinson metric as a function of the noise distribution. We also extend the continuity results of Arnold and Crauel [2] to the case of maps $g(x, y)$ which are not affine.

Elton[14, 15] has related results in a different context. He considered a stationary ergodic sequence $\left\{F_{n}\right\}$ of Lipschitz maps of a metric space $X$ into itself having almost surely a negative Lyapunov exponent (which he calls "contraction on average") and showed that the composition $F_{n} \ldots F_{1} x$ converges in distribution to a stationary ergodic process in $X$ independently of $x$. Related results are described in $[22,8,2]$. Theorem 5.1 shows that such Iterated Function Systems (IFS) have invariant measures which are continuous in the Hutchinson metric to deterministic perturbations to the maps $F_{n}$ in the Lipschitz metric or to random perturbations by additive i.i.d. noise in the fibre. In the uniformly contracting case this is implied by [7, Theorem 6.3 , page 360$]$. We generalise this result to the case of nonuniform contraction.

We establish notation and provide some background information in section 2 . The main results of this paper are stated in the theorems of section 3 and section 4 . Applications to IFS are given in section 5. The proofs are given in section 6 .

\section{Background and notation}

Let $(M, d)$ be a locally compact separable metric space and let $P(M)$ be the set of Borel probability measures on $M$. We let $L(M)$ denote the set of real-valued continuous maps $\psi$ on $M$ satisfying $\sup _{x \in M}|\psi(x)| \leq 1$ and $|\psi(x)-\psi(y)| \leq d(x, y)$ for all $x, y \in M$. We will define the Hutchinson metric $d_{H}[7$, page 349] on $P(M)$ by

$$
d_{H}(\nu, \tilde{\nu}):=\sup \left\{\int_{M} \psi d \nu-\int_{M} \psi d \tilde{\nu}: \psi \in L(M)\right\} .
$$

This gives the same topology on the $P(M)$ as the weak topology.

Remark 2.1 In Barnsley [7, page 349] the Hutchinson metric is defined for compact metric spaces $(M, d)$. To extend the definition to noncompact $M$ we require in essence that $\psi$ be uniformly continuous and bounded.

Let $X$ be a metric space equipped with a Borel measure $\mu$ and suppose that $f$ : $X \rightarrow X$ is a continuous map ergodic with respect to $\mu$. We call $(f, X, \mu)$ a dynamical 
system (in this paper $X$ will usually be a compact metric space $X$ ). Let Leb denote Lebesgue measure on $\mathbf{R}^{n}$ and let $\operatorname{Lip}\left(\mathbf{R}^{n}, \mathbf{R}^{n}\right)$ denote the set of Lipschitz maps from $\mathbf{R}^{n}$ to $\mathbf{R}^{n}$. Let $g(x,.) \in \operatorname{Lip}\left(\mathbf{R}^{n}, \mathbf{R}^{n}\right)$ for each $x \in X$ and suppose $g$ is continuous in both variables $x, y$ and Lipschitz in $y$.

Define the skew product

$$
T(x, y)=(f(x), g(x, y))
$$

On the space of maps $\{g(x,)\},. g(x,.) \in \operatorname{Lip}\left(\mathbf{R}^{n}, \mathbf{R}^{n}\right)$ we define the function $\|$.$\| by$

$$
\|g(x, .)\|:=\sup _{y \neq y^{\prime}} \frac{\left|g(x, y)-g\left(x, y^{\prime}\right)\right|}{\left|y-y^{\prime}\right|}
$$

and we put the metric

$$
d_{m}\left(g\left(x_{1}, .\right), g\left(x_{2}, .\right)\right):=\left\|g\left(x_{1}, .\right)-g\left(x_{2}, .\right)\right\|+\sup _{y \in \mathbf{R}^{n}}\left|g\left(x_{1}, y\right)-g\left(x_{2}, y\right)\right| .
$$

Define $g^{n}(x, y)$ by $T^{n}(x, y)=\left(f^{n}(x), g^{n}(x, y)\right)$ and assume that

$$
\lim _{n \rightarrow \infty} \frac{1}{n} \log \left\|g^{n}(x, .)\right\|
$$

exists for $\mu$ a.e. $x \in X$. This condition is satisfied if, for example, $\log ^{+}\|g(x,).\| \in$ $L^{1}(X, \mu)[20$, Theorem 5.4].

Since $(f, X, \mu)$ is assumed ergodic, if equation 2.4 holds then there exists $\lambda$ such that $\lambda(x)=\lambda$ for $\mu$ a.e. $x \in X$. We call $\lambda$ the maximal Lyapunov exponent in the fibre (with respect to $\mu$ ). We will say that the skew-product is weakly-stable if

$$
\lim _{n \rightarrow \infty} \frac{1}{n} \log \left\|g^{n}(x, .)\right\|=\lambda<0
$$

for $\mu$ a.e. $x \in X$.

For each $x$, the family of functions $\left\{a_{n}\right\}$ defined by $a_{n}(x)=\left\|g\left(f^{n} x,.\right)\right\|$ is subadditive, since $a_{n+l}(x) \leq a_{n}(x)+a_{l}(x)$ for all $n, l>0$ and hence by [20, Theorem 5.4, page $38]$

$$
\lim _{n \rightarrow \infty} \frac{1}{n} \log \left\|g^{n}(x, .)\right\|=\inf \frac{1}{n} \log \left\|g^{n}(x, .)\right\| .
$$

Thus if $g$ satisfies equation 2.5 for some $\lambda<0$ then given a sufficiently small $\epsilon>0$ there exists $\delta>0$ such that if $d_{m}(g(x,),. \tilde{g}(x,))<.\delta$ for $\mu$ a.e. $x \in X$ then

$$
\lim _{n \rightarrow \infty} \frac{1}{n} \log \left\|\tilde{g}^{n}(x, .)\right\|<\lambda+\epsilon<0
$$

for $\mu$ a.e. $x \in X$.

As a consequence of weak-stability, given $\epsilon>0$ there exists a measurable function $c: X \rightarrow \mathbf{R}^{+}$such that for $\mu$ a.e. $x \in X$, we have

$$
\left\|g^{n}(x, .)\right\|<c(x) e^{(\lambda+\epsilon) n}
$$

for all $n>0$.

We first state a useful variant of the Furstenberg-Kesten Theorem [16] due to Elton $[15]$. 
Proposition 2.2 [15, Prop 2,page 40]

(i) Let $Y$ be a complete separable locally compact metric space. Let $\left(F_{n}, n \geq 0\right)$ be a stationary process on an underlying probability space $(\Omega, \mathcal{F}, P)$ which takes values in the space of Lipschitz maps of $Y$ to itself. Suppose that $E \log ^{+}\left|F_{0}\right|<\infty$. Then there exists a measurable function $\lambda: \Omega \rightarrow \mathbf{R} \cup\{-\infty\}$ (called the Lyapunov exponent) with $\lambda^{+} \in L^{1}(P)$ such that

$$
\frac{1}{n} \log \left|F_{n} \ldots F_{1}\right| \rightarrow \lambda \text { a.s. }
$$

(ii) Furthermore, if $\left(F_{n},-\infty<n<\infty\right)$ extends $\left(F_{n}, n \geq 0\right)$ into the past, then for all $k$

$$
\begin{aligned}
& \lim _{n \rightarrow \infty} \frac{1}{n} \log \left|F_{k-1} \ldots F_{k-n}\right| \\
= & \lim _{n \rightarrow \infty} \frac{1}{n} \log \left|F_{n} \ldots F_{1}\right|=\lambda \text { a.s. }
\end{aligned}
$$

To form the natural extension of $(f, X, \mu)$ first we define a measure $\mu^{+}$on the set $X^{\mathbf{Z}^{+}}$by defining $\mu^{+}$on finite cylinder sets by $\mu^{+}\left(A_{0} \times A_{1} \times \ldots \times A_{n} \times X \times \ldots \times X \times \ldots\right)=$ $\mu\left(A_{0} \cap f^{-1} A_{1} \cap \ldots \cap f^{-n} A_{n}\right)$ and then extending the definition of $\mu^{+}$to $X^{\mathbf{Z}^{+}}$by Kolmogorov's extension theorem. We now define a measure $\bar{\mu}$ on $X^{\mathbf{Z}}$ in the following way. If $\left(\ldots \times X \times A_{-m} \times A_{-m+1} \times \ldots \times A_{0} \times \ldots \times A_{n} \times X \times \ldots \times X \ldots\right)$ is a finite cylinder set in $X^{\mathbf{Z}}$ then $\bar{\mu}\left(\ldots \times X \times A_{-m} \times A_{-m+1} \times \ldots \times A_{0} \times \ldots \times A_{n} \times X \times \ldots \times X \ldots\right)=\mu^{+}\left(h^{k}(\ldots \times\right.$ $\left.\left.X \times A_{-m} \times A_{-m+1} \times \ldots \times A_{0} \times \ldots \times A_{n} \times X \times \ldots \times X \ldots\right)\right)$ where $h$ is the shift map and $k$ is any positive integer larger than $m$. It is easy to check that $\bar{\mu}$ is well defined on cylinder sets and extends uniquely to a measure on $X^{\mathbf{Z}}$ (see Keller's book [18, Section 2.5] for an elegant exposition). .

The natural extension of $(f, X, \mu)$ is the dynamical system $(h, H, \bar{\mu})$ where $H=X^{\mathbf{Z}}$ and consists of bi-infinite sequences of the form $\left(\ldots, x_{-n} \ldots, x_{-1}, x_{0}, x_{1}, x_{2}, x_{3}, \ldots, x_{n}, \ldots\right)$ together with the invertible shift transformation $h\left(x_{i}\right)=\left(x_{i+1}\right)$. If $(X, d)$ is compact then we define the metric $d_{e}\left(\left(x_{i}\right),\left(x_{i}^{\prime}\right)\right):=\sum_{i=0}^{\infty} \frac{d\left(x_{i}, x_{i}^{\prime}\right)}{2^{n}}$ on $H$. We define the projection $\Pi_{0}: H \rightarrow X$ by $\Pi_{0}(w)=x_{0}$. From the definition of $\bar{\mu}$, it follows that $\bar{\mu} \cdot \Pi_{0}^{-1}=\mu$. The maps $h$ and $h^{-1}$ are ergodic with respect to $\bar{\mu}$ and $h$ is a homeomorphism with respect to $d_{e}$.

As a consequence of the modified Multiplicative Ergodic Theorem (Proposition $2.2)$, for $\bar{\mu}$ a.e. $w \in H$ there exists a $C(w)$ such that

$$
\left|g\left(\Pi_{0} h^{n}(w)\right) \ldots g\left(\Pi_{0} w\right)\right|<C(w) e^{(\lambda+\epsilon) n},\left|g\left(\Pi_{0}(w)\right) \ldots g\left(\Pi_{0} h^{-n} w\right)\right|<C(w) e^{(\lambda+\epsilon) n}
$$

for all $n \geq 0$.

One of the most important results which we shall use is the following proposition which shows that there are natural attractors in weakly-stable skew products. It is found in [15] and in a different guise in the work of [32], [10]. Campbell and Davies call the function $\Phi$ of the following proposition an "inertial function".

Proposition 2.3 [32, Theorem 1.4] [9, Theorem 5] Assume $(f, X, \mu)$ is invertible and the skew product $(x, y) \rightarrow(f(x), g(x, y))$ satisfies equation 2.5. Then there is a measurable function $\Phi: X \rightarrow \mathbf{R}^{n}$ such that $\Gamma$, the graph of $\Phi$, is invariant under $T,(T(x, \Phi(x))=(f(x), \Phi(f(x))))$. The graph $\Gamma$ (which may not be compact) is the support of an invariant measure $\Lambda$, and $(T, \Gamma, \Lambda)$ is ergodic (strong mixing, Bernoulli) if $(f, X, \mu)$ is ergodic (strong mixing, Bernoulli). Furthermore $\Gamma$ is attracting in the sense that for $\mu$ a.e. $x \in X, \lim _{n \rightarrow \infty}\left|\Pi_{y} \Gamma, \Pi_{y} T^{n}(x, y)\right|=0$ for every $y \in \mathbf{R}^{n}$. 
Corollary 2.4 If $(f, X, \mu)$ is not invertible then an easy argument shows that the conclusions of Proposition 2.3 apply to the skew product with the natural extension $(h, \bar{X}, \bar{m})$ of $(f, X, \mu)$ as the base transformation instead of $(f, X, \mu)$. There is a measurable function $\Phi: \bar{X} \rightarrow \mathbf{R}^{n}$ such that $\Gamma$, the graph of $\Phi$, is invariant under $h$. The graph $\Gamma$ (which may not be compact) is attracting and is the support of an invariant measure $\Lambda$ and is ergodic (mixing, Bernoulli) if $(h, H, \bar{\mu})$ is ergodic (mixing, Bernoulli).

\subsection{The structure of the invariant graph}

It is a natural question to ask what regularity properties the invariant graph of Proposition 2.3 possesses. Jaroslav Stark has interesting results on the regularity of such graphs in the case of uniform contraction [32]. These results have applications to the study of strange nonchaotic attractors. In the case of nonuniform contraction little can be claimed beyond the measurability of $\Phi$. We now give an example in which the base transformation is an analytic diffeomorphism and the function $g(x, y)$ is smooth yet the map $\Phi: X \rightarrow \mathbf{R}^{n}$ of Proposition 2.3 is not even integrable with respect to $\mu$.

Example 2.5 Suppose $(f, X, \mu)$ is the Arnold Cat map i.e. the hyperbolic automorphism

$$
\left(\begin{array}{ll}
2 & 1 \\
1 & 1
\end{array}\right)
$$

of the two-dimensional torus which has Lebesgue measure on $T^{2}$ as an invariant measure. We consider $T^{2}$ to be $[0,1] \times[0,1]$ with opposite sides identified. The origin $(0,0)$ is a fixed point. For small $\epsilon, \delta>0$ we define $g(x, y)=2 y+\epsilon$ for $d(x,(0,0))<\delta$ and $g(x, y)=10^{-6} y+\epsilon$ for $x$ such that $d(x,(0,0))>2 \delta$. We define $g(x, y)$ smoothly as a function of $x$ in the region $\delta \leq d(x,(0,0)) \leq 2 \delta$ so that $\frac{g(x, y)}{y} \leq 2+\epsilon$. If $\delta$ is sufficiently small then this dynamical system has a negative maximal Lyapunov exponent and hence an invariant attracting graph given by a function $\Phi: T^{2} \rightarrow \mathbf{R}$. Since the graph is invariant $\Phi(x) \geq \epsilon$ for all $x$ and so $\Phi(x) \geq 2^{n} \epsilon$ if $x \in A(n):=\left\{x: d\left(f^{-j} x,(0,0)\right)<\delta, j=\right.$ $0,1, \ldots, n\}$. The set $A(n)$ has measure greater than $\delta \lambda^{-n}$ where $\lambda:=\frac{\sqrt{5}+1}{2}$ is the maximal eigenvalue of $f$. For any $n, \int_{T^{2}} \Phi(x) d x \geq \int_{A(n)} 2^{n} \epsilon \geq\left(\frac{2}{\lambda}\right)^{n} \epsilon \delta$. Thus $\Phi: T^{2} \rightarrow \mathbf{R}$ is not integrable with respect to Lebesgue measure. Note that this nonintegrability is persistent under small Lipschitz perturbations of $g(x, y)$.

It is clear that in general we cannot hope for stability of attractors $\Gamma$ in the Hausdorff metric if the contraction in the fibre is not uniform.

\section{Deterministic perturbations}

Suppose that $T(x, y)=(f(x), g(x, y))$ satisfies equation 2.5. We now consider the effect of perturbing $g(x,$.$) in the metric d_{m}$. As we have discussed if $d_{m}(g(x,),. \tilde{g}(x,)$. is sufficiently small for $\mu$ a.e. $x \in X$ then $\tilde{T}(x, y):=(f(x), \tilde{g}(x, y))$ also satisfies equation 2.5 and hence the conclusions of Proposition 2.3 hold. We denote the invariant graph and invariant measure on the graph for $\tilde{T}(x, y)=(f(x), \tilde{g}(x, y))$ by $\tilde{\Gamma}$ and $\tilde{\Lambda}$ respectively. 
Theorem 3.1 Suppose $T(x, y)=(f(x), g(x, y))$ satisfies equation 2.5. Let $\epsilon>0$. Then there exists $\delta>0$ such that if $d_{m}(g(x,),. \tilde{g}(x,))<.\delta$ for $\mu$ a.e. $x \in X$, then for $\mu$ a.e. $x \in X$ for all $y \in \mathbf{R}^{n}$

$$
d\left(T^{n}(x, y), \tilde{T}^{n}(x, y)\right)<\epsilon
$$

except for at most a fraction $\epsilon$ of times $n$.

Furthermore $d_{H}(\Lambda, \tilde{\Lambda})<\epsilon$ (Hutchinson metric).

Remark 3.2 If the base transformation $(f, X, \mu)$ is uniquely ergodic then the conclusion of Theorem 3.1 may be strengthened to $d\left(T^{n}(x, y), \tilde{T}^{n}(x, y)\right)<\epsilon$ for all $n$.

\section{Random perturbations}

In this section we consider random perturbations of systems of form 2.3 by additive noise taking values in the fiber $\mathbf{R}^{n}$.

Suppose we perturb the system in the fibre by bounded i.i.d. noise (not necessarily mean zero) so that

$$
(x, y) \rightarrow(f(x), g(x, y)) \rightarrow(f(x), g(x, y)+\sigma)
$$

where $\sigma$ comes from a bounded i.i.d. noise process taking values in a compact set $\Delta$, which may be discrete or contain open sets.

The noise process is modelled in the usual way. Suppose that $\Delta \subset \mathbf{R}^{n}$ is the compact support of a probability measure $\nu^{\prime}$ which gives the distribution of the noise. Let $s=\max _{x \in \Delta}|x|$.

We equip $\Omega \equiv \Delta^{\mathbf{Z}}$ with the product topology and the product measure $\nu$ induced from $\nu^{\prime}$. We form the Bernoulli shift,

$$
S: \Omega \rightarrow \Omega, S(\sigma)_{n}=\sigma_{n+1} .
$$

The transformation $F \equiv(f \times S): X \times \Omega \rightarrow X \times \Omega$ is ergodic with respect to the measure $\mu \times \nu$ since $(f, X, \mu)$ is ergodic and $(S, \Omega, \nu)$ is a Bernoulli shift.

Finally we define

$$
G(x, \sigma, y)=\left(f(x), S \sigma, g(x, y)+\sigma_{0}\right)
$$

Let $\Pi_{x}(x, \sigma, y)=x$ denote the projection onto the $x$-component and similarly let $\Pi_{y}$ denote the projection onto the $\mathbf{R}^{n}$-component. Let $\Pi(x, \sigma, y)=(x, y)$ denote the projection onto the $X$ and $\mathbf{R}^{n}$ components.

We write the system given by equation 4.6 as $G(w, y)=(F(w), \hat{g}(w, y))$ where $w=(x, \sigma), F(w)=(f x, S \sigma)$ is a continuous map of the compact metric space $X \times \Omega$ and $\hat{g}(w, y)=\tilde{g}(x, y)+\sigma_{0}$.

As a modification of proposition 2.3 we have the following description of the perturbed dynamics.

Corollary 4.1 (to prop 2.3) There is a function $\Phi: X \times \Omega \rightarrow \mathbf{R}^{n}$ such that $\Gamma$, the graph of $\Phi$, is invariant under $G,(G(w, \Phi(w))=(F(w), \Phi(F(w))$ ). The graph $\Gamma$ (which may not be compact) is the support of an invariant measure $\Lambda$, and $(G, \Gamma, \Lambda)$ is ergodic (strong mixing, Bernoulli) if $(f, X, \mu)$ is ergodic (strong mixing, Bernoulli). Furthermore $\Gamma$ is attracting in the sense that for $\mu \times \nu$ a.e. $w \in X \times \Omega, \lim _{n \rightarrow \infty}\left|\Pi_{y} \Gamma, \Pi_{y} G^{n}(w, y)\right|=$ 0 for every $y \in \mathbf{R}^{n}$. 
Corollary 4.2 If $\Delta$ has a non-empty interior then for $\mu$ a.e. $x, \Gamma_{x}$, the fibre which projects to $\{x\}$, will contain sets of positive Lebesgue measure [35, Proposition 1.2].

Corollary 4.3 There exists a unique positive Borel measure $K$ on $X \times \mathbf{R}^{n}$ such that for any continuous $\phi: X \times \mathbf{R}^{n} \rightarrow \mathbf{R}$, for $\mu \times$ Leb a.e. $(x, y) \in X \times \mathbf{R}^{n}$,

$$
\lim _{n \rightarrow \infty} \frac{1}{n} \sum_{i=0}^{n-1} \phi \cdot \Pi\left(G^{n}(x, \sigma, y)\right)=\int_{X \times \mathbf{R}^{n}} \phi d K
$$

with probability one.

For a fixed map $T(x, y)=(f(x), g(x, y))$ arising from an ergodic base transformation $(f, X, \mu)$ and cocycle $g$ we call $\Lambda$ of Corollary 4.3 the stochastic measure associated to $\nu$.

Theorem 4.4 Let $\nu$ be a compactly supported Borel measure on $\mathbf{R}^{n}$ and which determines the distribution of the noise process i.e. the random variables $\sigma_{i}$ take values in the set $\Delta=\operatorname{Support}(\nu)$. Given $\epsilon>0$ there exists $\delta>0$ such that if $d_{H}(\nu, \tilde{\nu})<\delta$ then $\tilde{\Lambda}$, the stochastic measure corresponding to $\tilde{\nu}$ satisfies $d_{H}(\Lambda, \tilde{\Lambda})<\epsilon$.

Remark 4.5 Thus there is continuity of averaged observables as a function of the noise distribution.

We now relax the assumption that the noise process is i.i.d. or even stationary. In this context we have the following result.

Theorem 4.6 Suppose $(x, y) \rightarrow(f(x), g(x, y))$ satisfies equation 2.5. Let $\epsilon>0$. Then there exists $\delta>0$ such that if $\max _{s \in \Delta}|s|<\delta$ then for $\mu$ a.e. $x \in X$, for all $y \in \mathbf{R}^{n}$, for all $\sigma \in \Delta^{\mathbf{Z}}$

$$
d\left(T^{n}(x, y), G^{n}(x, \sigma, y)\right)<\epsilon
$$

except for at most a fraction $\epsilon$ of times $n$.

\section{$5 \quad$ An application to IFS}

We briefly give an application of these ideas to IFS. Further results related to stochastic bifurcation theory will be given in a forthcoming paper.

\subsection{Iterated Function Systems}

Suppose $\left\{F_{1}, \ldots, F_{n}\right\}$ is a set of Lipschitz maps of $\mathbf{R}^{n}$ with the map $F_{i}$ having Lipschitz constant $C_{i}$. We form the IFS $\left(F_{1}, \ldots, F_{n}: p_{1}, \ldots, p_{n}\right)$ by choosing $F_{i}$ with probability $p_{i}$ $\left(\sum_{i=1}^{n} p_{i}=1\right)$. Assume we have 'contraction on average' i.e.

$$
\sum_{i=1}^{n} p_{i} \log \left|C_{i}\right|<0
$$

This system is a skew-product whose base transformation is the Bernoulli shift $(S w)_{0}=w_{1}$ on $\Omega \equiv\{1, \ldots, n\}^{\mathbf{Z}}$ with the product measure $\nu$ induced on cylinder sets 
from the measure $\nu^{\prime}(i)=p_{i}$ i.e. the skew-product $T(w, y)=\left(S w, F_{w_{0}}(y)\right)$, where $w \in \Omega, y \in \mathbf{R}^{n}$ and $(S, \Omega, \nu)$ is Bernoulli.

Contraction on average implies weak-stability of the skew-product so there exists an invariant attracting set $\Gamma$ which has an invariant measure $\Lambda$, and $\Gamma$ is the graph of a function $\Phi$ of $\Omega=\{1, \ldots, n\}^{\mathbf{Z}}$. Thus $\Gamma$ has form $(w, \Phi(w))$. Suppose now that we perturb $F_{i}$ to $\tilde{F}_{i}, i=1, \ldots, n$, to form the perturbed skew-product $\tilde{T}(w, y)=\left(S w, \tilde{F}_{w_{0}}(y)\right)$.

Theorem 5.1 Given $\epsilon>0$ there exists $\delta>0$ such that if $d_{m}\left(F_{i}, \tilde{F}_{i}\right)<\delta$ for all $i=1, \ldots, n$ then for $\nu$ a.e. $\omega \in \Omega$, for all $y \in \mathbf{R}^{n}$

$$
\left|\Pi_{y} T^{n}(\omega, y), \Pi_{y} \tilde{T}^{n}(\omega, y)\right|<\epsilon
$$

except for at most a fraction $\epsilon$ of times $n$.

Furthermore $d_{H}(\Lambda, \tilde{\Lambda})<\epsilon$.

\section{Proof of the main theorems}

Proof of Theorem 3.1

First we will prove the existence of the graph $\Phi$. To simplify notation assume that $f$ is invertible. Note that if $f$ is ergodic and invertible then $f^{-1}$ is also ergodic. Hence $f^{-1}$ is ergodic with respect to $\mu$ and has the same spectrum of Lyapunov exponents in the fibre by 2.2. Thus if we define

$$
\psi_{n}(x, y)=g^{n}\left(f^{-n} x, y\right)
$$

then

$$
\lim _{n \rightarrow \infty} \frac{1}{n} \log \left\|\psi_{n}(x, .)\right\|=\lambda<0
$$

for $\mu$ a.e. $x \in X$. Hence given $\epsilon>0$, for $\mu$ a.e. $x \in X, \exists l(x) \in \mathbf{N}$ such that

$$
\left\|\psi_{n}(x, .)\right\|<e^{n(\lambda+\epsilon)} \quad \forall n \geq l(x) .
$$

Thus given $\epsilon>0$, for $\mu$ a.e. $x \in X$, there exists $c(x) \in \mathbf{N}$ such that

$$
\left\|\psi_{n}(x, .)\right\|<c(x) e^{n(\lambda+\epsilon)} \quad \forall n \geq 0 .
$$

We will use this fact to show that for fixed $y \in \mathbf{R}^{n}$ for $\mu$ a.e. $x \in X,\left\{\psi_{l}(x, y)\right\}$ is a Cauchy sequence. Let

$$
K(y)=\sup _{x \in X}|y-g(x, y)|
$$

and note that for $y$ fixed $K(y)$ is finite as $X$ is compact and $g$ continuous. Given any $\epsilon^{\prime}>0$, choose $l^{*}(x)$ sufficiently large that

$$
K(y) c(x) \sum_{j=l^{*}(x)}^{\infty} e^{j(\lambda+\epsilon)}<\epsilon^{\prime}
$$

Then if $m>l>l^{*}(x)$,

$$
\left|\psi_{m}(x, y)-\psi_{l}(x, y)\right| \leq K(y) \sum_{j=l}^{\infty}\left\|\psi_{j}(x, .)\right\|
$$




$$
\leq K(y) c(x) \sum_{j=l}^{\infty} e^{j(\lambda+\epsilon)}<\epsilon^{\prime} .
$$

To see this note that

$$
\left|\psi_{m}(x, y)-\psi_{l}(x, y)\right|=\left|\psi_{m}(x, y)-\psi_{m-1}(x, y)+\cdots+\psi_{l+1}(x, y)-\psi_{l}(x, y)\right| .
$$

Note that applying $T$ once to $\left(f^{-k} x, y\right)$, gives $T\left(f^{-k} x, y\right)=\left(f^{-(k-1)} x, g\left(f^{-k} x, y\right)\right)$. Thus $\psi_{k}(x, y)-\psi_{k-1}(x, y)=\psi_{k-1}\left(x, g\left(f^{-k} x, y\right)\right)-\psi_{k-1}(x, y)$. As a result,

$$
\left|\psi_{k-1}(x, y)-\psi_{k}(x, y)\right| \leq|| \psi_{k-1}(x, .)||\left|y-g\left(f^{-k} x, y\right)\right|
$$

Hence

$$
\begin{aligned}
\left|\psi_{m}(x, y)-\psi_{l}(x, y)\right| & \leq\left|\psi_{m}(x, y)-\psi_{m-1}(x, y)\right|+\cdots+\left|\psi_{l+1}(x, y)-\psi_{l}(x, y)\right| \\
& =\sum_{j=l+1}^{m}\left|\psi_{j}(x, y)-\psi_{j-1}(x, y)\right| \\
& \leq \sum_{j=l+1}^{m}\left\|\psi_{j-1}(x, .)\right\|\left|y-g\left(f^{-j} x, y\right)\right| \\
& \leq \sum_{j=l+1}^{\infty}\left\|\psi_{j-1}(x, .)\right\| K(y) .
\end{aligned}
$$

Thus

$$
\begin{aligned}
\left|\psi_{m}(x, y)-\psi_{l}(x, y)\right| & \leq K(y) \sum_{j=l+1}^{\infty}\left\|\psi_{j-1}(x, .)\right\| \\
& <K(y) c(x) \sum_{j=l+1}^{\infty} e^{(j-1)(\lambda+\epsilon)} \\
& =K(y) c(x) \sum_{j=l}^{\infty} e^{j(\lambda+\epsilon)}<\epsilon^{\prime}
\end{aligned}
$$

as $l>l^{*}(x)$. Thus $\left\{\psi_{m}(x, y)\right\}$ is a Cauchy sequence for every $y \in \mathbf{R}^{n}$.

Define

$$
\Phi(x)=\lim _{m \rightarrow \infty}\left\{\psi_{m}(x, 0)\right\} .
$$

Note that each $\psi_{m}(x, 0)$ is continuous (as $x$ varies) and hence measurable. As the limit of a sequence of measurable functions is measurable, $\Phi$ is measurable. Now, since

$$
T\left(x, \psi_{l}(x, 0)\right)=\left(f(x), \psi_{l+1}(f(x), 0)\right)
$$

we see that

$$
T(x, \Phi(x))=(f(x), \Phi(f(x))
$$

and hence $\Gamma$, the graph of $\Phi$ is invariant under $T$. Furthermore, by construction

$$
\lim _{m \rightarrow \infty}\left|g^{m}(x, y)-g^{m}(x, 0)\right|=0
$$

for all $y \in \mathbf{R}^{n}$, so $\Gamma$ is an attracting invariant set. This is because

$$
\left|g^{m}(x, y)-g^{m}(x, 0)\right| \leq\left\|g^{m}(x, .)\right\||y|
$$


and $\left\|g^{m}(x,).\right\| \rightarrow 0$ as $m \rightarrow \infty$. It also follows easily that $\Gamma$ attracts orbits asymptotically at an exponential rate.

The mixing properties of the base transformation $(f, X, \mu)$ lift to the transformation $(T, \Gamma, \Lambda)$ when $\Lambda:=\mu \circ(I d \times \Phi)^{-1}$ where $\operatorname{Id}(x)=x$ is the identity mapping on $X$.

If $f$ is not invertible then an argument involving the natural extension yields Corollary 2.4 with no essential modification to the proof, though the notation is more cumbersome. Given $(f, X, \mu)$ we form the natural extension $(h, H, \bar{\mu})$ and define $\bar{g}(\bar{x}, y):=$ $g\left(x_{0}, y\right)$ where $\bar{x}=\left(x_{i}\right) \in H \cdot \bar{g}(\bar{x}, y)$ is continuous in $\bar{x}$ with respect to the $d_{e}$ metric and Lipschitz in $y$. The skew product system $\bar{T}(\bar{x}, y):=(h(\bar{x}), \bar{g}(y))$ satisfies equation 2.5 and the argument proceeds exactly as that given above in which $f$ was assumed invertible.

We now continue with the proof of Theorem 3.1 under the assumption that $f$ is invertible. Let $\epsilon>0$. We have already seen that for any $0<\zeta<\epsilon$, for $\mu$ a.e. $x \in X$, $\exists l(x) \in \mathbf{N}$ such that

$$
\left\|\psi_{n}(x, .)\right\|<e^{n(\lambda+\zeta)} \quad \forall n \geq l(x)
$$

Define

$$
K(n):=\left\{x \in X:\left\|\psi_{l}(x, .)\right\| \leq e^{(\lambda+\zeta) l} \quad \forall l \geq n\right\}
$$

Note that $\lim _{n \rightarrow \infty} \mu(K(n))=1$. So given any $\epsilon>0$, there exists $N \in \mathbf{N}$ such that $\mu(K(N))>1-\epsilon$. Fix $N$ with this property and choose $\delta>0$ such that

$$
\delta\left(\sum_{i=0}^{N-1}\left\|\psi_{i}(x, .)\right\|+\sum_{i=N}^{\infty} e^{(\lambda+\zeta) i}\right)<\epsilon .
$$

A crucial observation is that

$$
\psi_{n}(x, y)=\psi_{n-1}\left(x, g\left(f^{-n} x, y\right)\right) .
$$

Thus

$$
\begin{aligned}
\left|\psi_{n}(x, y)-\tilde{\psi}_{n}(x, y)\right| & =\mid \psi_{n-1}\left(x, g\left(f^{-n} x, y\right)\right)-\psi_{n-1}\left(x, \tilde{g}\left(f^{-n} x, y\right)\right) \\
& +\psi_{n-1}\left(x, \tilde{g}\left(f^{-n} x, y\right)\right)-\tilde{\psi}_{n-1}\left(x, \tilde{g}\left(f^{-n} x, y\right)\right) \mid
\end{aligned}
$$

Now

$$
\left|\psi_{n-1}\left(x, g\left(f^{-n} x, y\right)\right)-\psi_{n-1}\left(x, \tilde{g}\left(f^{-n} x, y\right)\right)\right| \leq\left\|\psi_{n-1}(x, .)\right\| \delta
$$

since $d_{m}(g(x,),. \tilde{g}(x,))<.\delta$.

Using the above and proceeding inductively, we find that

$$
\left|\psi_{n}(x, y)-\tilde{\psi}_{n}(x, y)\right| \leq \delta \sum_{i=0}^{n-1}\left\|\psi_{i}(x, .)\right\|
$$

Now, if $x \in K(N)$, taking the limit of both sides gives

$$
\begin{aligned}
\lim _{n \rightarrow \infty}\left|\psi_{n}(x, y)-\tilde{\psi}_{n}(x, y)\right| & \leq \lim _{n \rightarrow \infty} \delta \sum_{i=0}^{n-1}\left\|\psi_{i}(x, .)\right\| \\
& \leq \delta\left(\sum_{i=0}^{N-1}\left\|\psi_{i}(x, .)\right\|+\sum_{i=N}^{\infty} e^{(\lambda+\zeta) i}\right)<\epsilon
\end{aligned}
$$


i.e. $|\Phi(x)-\tilde{\Phi}(x)|<\epsilon$. Note that this holds on a set of measure at least $1-\epsilon$ (the measure of $K(N))$, so by ergodicity and the fact that $\Gamma$ is attracting $\left|T^{n}(x, y)-\tilde{T}^{n}(x, y)\right|<\epsilon$ for all but at most a fraction $\epsilon$ of times $n$.

Suppose now that $\psi \in L(M)$, then by ergodicity and the fact that $\Gamma$ is attracting for $\mu$ a.e. $x$, for all $y \in \mathbf{R}^{n}$

$$
\begin{gathered}
\lim _{n \rightarrow \infty} \sum_{j=0}^{n-1} \psi\left(T^{j}(x, y)\right) \\
=\lim _{n \rightarrow \infty} \sum_{j=0}^{n-1} \psi\left(T^{j}(x, \Phi x)\right) \\
=\int_{\Gamma} \psi d \Lambda
\end{gathered}
$$

Since $\left|T^{n}(x, y)-\tilde{T}^{n}(x, y)\right|<\epsilon$ for all but at most a fraction $\epsilon$ of times $n$ we see that

$$
\lim _{n \rightarrow \infty} \sum_{j=0}^{n-1}\left|\psi\left(T^{j}(x, y)\right)-\psi\left(\tilde{T}^{j}(x, y)\right)\right|
$$

is less than $2 \epsilon$ for all $\psi \in L(M)$ and hence $d_{H}(\Lambda, \tilde{\Lambda})<2 \epsilon$.

This implies the theorem in the case that $f$ is invertible. If $(f, X, \mu)$ is not invertible we form the natural extension $(h, H, \bar{\mu})$ and repeat the argument above with the natural extension as the base transformation and fibre map $\bar{g}(\bar{x}, y):=g\left(x_{0}, y\right)$. The supposition that $d_{m}(g(x,),. \tilde{g}(x,))<.\delta$ for $\mu$ a.e. $x$ implies that the corresponding maps $\bar{g}(\bar{x},$.$) ,$ $\tilde{\bar{g}}(\bar{x},$.$) satisfy d_{m}(\bar{g}(\bar{x},),. \tilde{\bar{g}}(\bar{x},))<.\delta$ for $\bar{\mu}$ a.e. $\bar{x}$. Define $T_{h}(\bar{x}, y)=(h(\bar{x}), \bar{g}(\bar{x}, y))$ and similarly $\tilde{T}_{h}(\bar{x}, y)=(h(\bar{x}), \tilde{\bar{g}}(\bar{x}, y))$. The same proof given above shows that if $\delta$ is sufficiently small then for $\bar{\mu}$ a.e. $\bar{x}, d\left(T_{h}^{n}(\bar{x}, y), \tilde{T}_{h}{ }^{n}(\bar{x}, y)\right)<\epsilon$ except for at most a fraction $\epsilon$ of times $n$. As a consequence of the definition of $T_{h}$ and the measure $\bar{\mu}$ this implies Theorem 3.1 in the case that $(f, X, \mu)$ is not invertible.

To prove Corollary 4.1 note that i.i.d. additive noise in the fibre will not affect the fact that the system has a negative maximal Lyapunov exponent in the fibre when the system is written as a skew product over the dynamical system $F:=(f \times S): X \times \Omega \rightarrow$ $X \times \Omega$ with invariant measure $\mu \times \nu$ if the unperturbed skew product mapping $T$ had a negative maximal Lyapunov exponent in the fibre. Since $(S, \Omega, \nu)$ is a Bernoulli dynamical system $(G, \Gamma, \Lambda)$ is ergodic (strong mixing, Bernoulli) if $(f, X, \mu)$ is ergodic (strong mixing, Bernoulli).

The proof of Theorem 4.4 and Theorem 4.6 proceed in the same way with the base transformation $G(\omega, y)=(F(\omega), \hat{g}(\omega, y))$ being used instead of $f$. Theorem 5.1 is proved by noting that the perturbed system $\tilde{T}(\omega, y):=\left(S \omega, \tilde{F}_{\omega_{0}}(y)\right)$ also possesses an invariant graph $\tilde{\Phi}: \Omega \rightarrow \mathbf{R}^{n}$ if $\delta$ is sufficiently small. By writing $\tilde{F}_{i}=F_{i}+\left(\tilde{F}_{i}-F_{i}\right)$ we may use Theorem 4.6 to conclude that if $\delta>0$ is sufficiently small then $|\tilde{\Phi}(\omega)-\Phi(\omega)|<\epsilon$ except for a set of $\omega \in \Omega$ of measure at most $\epsilon$. The same arguments used in the proof of Theorem 3.1 then yield the conclusions of Theorem 5.1.

\section{Acknowledgements}

It is a pleasure to acknowledge helpful discussions with Jaroslav Stark, Pete Ashwin and Kevin Campbell. 


\section{References}

[1] J.C. Alexander, I. Kan, J.A. Yorke and Zhiping You. Riddled Basins. Int. Journal of Bifurcations and Chaos, 2:795-813, 1992.

[2] Arnold \& Crauel. Iterated Function Systems and Multiplicative Ergodic Theory. Stochastic Flows-Proceedings of a conference in Charlotte, Virginia M. Pinsky 86 V. Wihstutz (editors):284-305, Birkhauser Verlag, 1990.

[3] P. Ashwin and E. Stone. On the influence of noise near blowout bifurcation. to appear Phys. Rev. E., 1997.

[4] P. Ashwin, J. Buescu and I.N. Stewart. Bubbling of attractors and synchronisation of oscillators. Phys. Lett. A 193:126-139, 1994.

[5] P. Ashwin, J. Buescu and I.N. Stewart. From attractor to chaotic saddle: a tale of transverse instability. Nonlinearity 9:703-737, 1996.

[6] P. Ashwin, P.J. Aston and M. Nicol. On the unfolding of a blowout bifurcation. Physica D 111:81-95, 1998.

[7] Michael F. Barnsley. Fractals Everywhere. Second Edition, Academic Press, 1993.

[8] M. A. Berger Random Affine Iterated Function Systems: Mixing and Encoding Stochastic Flows-Proceedings of a conference in Charlotte, Virginia M. Pinsky 86 V. Wihstutz (editors):315-345, Birkhauser Verlag, 1990.

[9] K. M. Campbell Observational noise in skew product systems Physica D, to appear 1997.

[10] K. M. Campbell and M. E. Davies The existence of inertial functions in skew product systems Nonlinearity,Vol 9, 3:801-819, 1996.

[11] A. Cenys and H. Lustfeld. Statistical properties of the noisy on-off intermittency. J. Phys. A Math. Gen. 29:11-20, 1996.

[12] K.M. Cuomo and A.V. Oppenheim Circuit implementation of synchronized chaos with applications to communication Phys. Rev. Lett. 71:65-68, 1993.

[13] J. Elton and J. Ezzine. Weak Convergence and Generalized Stability for Solutions to Random Dynamical Systems. Stochastic Flows-Proceedings of a conference in Charlotte, Virginia M. Pinsky $E^{3}$ V. Wihstutz (editors):307-314, Birkhauser Verlag, 1990.

[14] J. H. Elton. An ergodic theorem for iterated maps. Ergod. Th. ES Dynam. sys. 7:481-488, 1987.

[15] J. H. Elton. A multiplicative ergodic theorem for Lipschitz maps. Stochastic Processes and their Applications 34:39-47, 1990.

[16] H. Furstenberg and H. Kesten. Products of random matrices. Ann. Math. Stat.31: 457-469 (1960) 
[17] A. Katok and B. Hasselblatt. Introduction to the modern theory of dynamical systems. Encyclopedia of mathematics and its applications 54 Cambridge University Press, 1995.

[18] G. Keller. Equilibrium states in ergodic theory LMSST 42 Cambridge University Press, 1998.

[19] I. Kocarev and U. Parlitz. General approach for chaotic synchronization with applications to communication Phys. Rev. Lett. 74:5028-5031 , 1995.

[20] U. Krengel. Ergodic Theorems. de Gruyter Studies in Mathematics 6, Walter de Gruyter, 1985.

[21] F. Ledrappier and L-S. Young. Stability of Lyapunov exponents. Ergod. Th. \&6 Dynam. Sys. 11:469-484 (1991)

[22] A. Mukherjea Distribution of Markov Processes Generated by I.I.D Random Matrices. Stochastic Flows-Proceedings of a conferencein Charlotte, Virginia M. Pinsky $\&$ V. Wihstutz (editors):171-199, Birkhauser Verlag, 1990.

[23] V.I. Oseledec. A multiplicative ergodic theorem: Liapunov characteristic numbers for dynamical systems. Trans. Mosc. Math. Soc. 19:197-231, 1968.

[24] E. Ott, J.C. Sommerer, J.C. Alexander, I. Kan and J.A. Yorke. Scaling behaviour of chaotic systems with riddled basins. Phys. Rev. Lett. 71:4134-4137, 1993.

[25] E. Ott, J.C. Sommerer, J.C. Alexander, I. Kan and J.A. Yorke. A transition to chaotic attractors with riddled basins. Physica D 76: 384-410, 1994.

[26] E. Ott, J.C. Sommerer. Blowout bifurcations: the occurrence of riddled basins and on-off intermittency. Phys. Lett. A 188: 39-47, 1994.)

[27] L.M. Pecora and T.L. Carroll Synchronisation in Chaotic Systems Phys. Rev. Lett. 64:821-824, 1990.

[28] N. Platt, E. A. Spiegel and C. Tresser. Effects of additive noise on on-off intermittency Phys. Rev. Lett. 72:3498-3501, 1994.

[29] Mark Pollicott. Lectures on ergodic theory and Pesin theory on compact manifolds. LMS Lecture Note Series 180, CUP, 1993.

[30] W. Rudin. Real and Complex Analysis. Third Edition, McGraw Hill, (1987).

[31] D. J. Rudolph. Classifying the compact extensions of a Bernoulli shift. J. Analyse Math. 34:36-59, 1978.

[32] Jaroslav Stark. Regularity of invariant graphs for forced systems. Preprint, August, 1996.

[33] Jaroslav Stark. Invariant graphs for forced systems. Preprint, October, 1996.

[34] S. C. Venkatarami, B. R. Hunt and E. Ott. Bubbling transition. Phys Rev E 54:1346-60,1996.

[35] L. S. Young. Stochastic stability of hyperbolic attractors. Ergod. Th. E Dynam. Sys. 16:651-662 (1996) 